\title{
Robust Coplanar Bus with Unique Modular Design for Quantum-dot Cellular Automata
}

\author{
Mridul Kumar Mathur', Waseem H. Wani ${ }^{2}$, Z. A. Bangi ${ }^{3 *}$, M. Tariq Banday ${ }^{3}$, \\ S. Umira R. Qadri ${ }^{3}$ and Debashis $\mathrm{De}^{4}$ \\ 'Department of Computer Science, LMCST, Jodhpur - 342001, Rajasthan, India; mridulmathur005@gmail.com \\ 2Department of Computer Application, Jodhpur National University, Jodhpur - 342003, Rajasthan, \\ India; waseem_hu2003@yahoo.com \\ ${ }^{3}$ Department of Electronics and IT, University of Kashmir, Srinagar - 190006, Jammu and Kashmir, \\ India; zubifayaz@gmail.com,sgrmtb@gmail.com, umirariyaz@gmail.com \\ ${ }^{4}$ Department of Computer Science and Engineering, West Bengal University of Technology, \\ Kolkata - 700064, West Bengal, India; dr.debashis.de@gmail.com
}

\section{Abstract}

Objective: The proposed designs have been implemented in order to exploit the properties of Quantum Dot Cellular Automata majority voting to consent a modular robust crossing of wires. Methods/Statistical Analysis: Three structures namely $4 \times 4,5 \times 5$ and $8 \times 8$ coplanar buses with unique modular design in quantum dot cellular automata have been presented and the operation of these structures has been tested by QCA Designer Simulation Tool. These unique structures present a modus operandi for designing the modified robust coplanar bus with unique modular approach in nanotechnology. Findings: The proposed modular coplanar structures allow number of coplanar wires to cross without signal degradation and signal loss until information propagates the output. Compared to the reported literature, the proposed design has following advantages;

- Unique modular approach in which wires can be intersected on the single layer without any intrusion or crosstalk.

- High output polarity, fault tolerant capabilities, modular approach and robustness which makes it more efficient without any interference;

- Use of sequential clock which makes it easier to understand and manufacture;

- The signal and its counterpart can be simply taken out together due to the alternating polarization.

- Proper use of clocking phase pairs in a traversed manner in order to permit the accurate propagation of signals.

- Cells are evenly disseminated into the clocking zones which are implemented that the unusable spaces can be as small as feasible.

- It has been concluded that it is easy to increase the number of inputs by adding the suitable layers with proposed unique modular approach.

Application/Improvements: Starting from lowest number of inputs, it is easy to extend the proposed designs to large and then larger number of inputs by adding suitable layers which will prove efficient to tolerate various faults during the manufacturing process.

Keywords: Coplanar Crossing, Fault Tolerant Computing, Modular, Nanotechnology, Quantum-dot Cellular Automata, Robust 


\section{Introduction}

Quantum-dot Cellular Automata (QCA) proposed by Lent et. al ${ }^{12}$ in the beginning,is a novel technique for designing digital circuits at nano-scale in which the binary information is stowed as one of the two diagonal depictions of electron pair in quantum-dot containing at least four energy states as shown in Figure 1. Conventional CMOS technology uses range of voltages or currents to represent binary values. QCA uses the advantages of quantum-mechanical effects in order to decrease the size of digital circuits and works on high speed at very low power levels. The main advantages of QCA are simplicity, low power delay product and extremely high logic integration resulting from small size of dots?

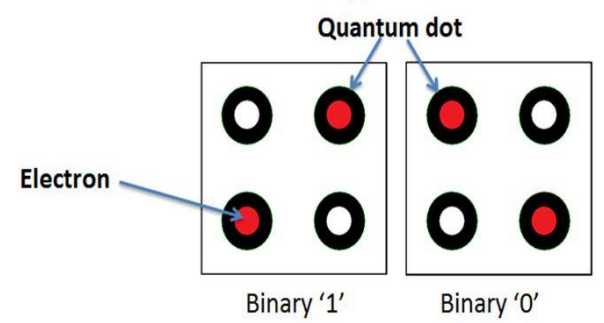

Figure 1. Binary 1 and 0 Representations and Cell Polarizations.

Quantum dots in QCA interact using Columbic contact and the quantum-mechanical tunneling where electrons tunnel between the dots, however cannot evacuate the cell. In the truancy of an external electrostatic effect, columbic repulsion will force the electrons to line up on the differing corners of the dots, if two mobile electrons are positioned in the ground state of the cell[ ${ }^{4-0}$. Arrays of these coupled quantum dots are used to accomplish convenient computations and to form Boolean function?. QCA cells need to line up precisely at nano-scales to offer accurate functionality as the industrial imperfections and misalignment plays an important role in the quality of digital designs

Clocking mechanism is the main feature in QCA which is used in order to transfer data, which operates by increasing or decreasing the tunneling barriers between the electrons and comprises of four stages: Hold, Switch, Release and Relax (Null) as in Figure 2.

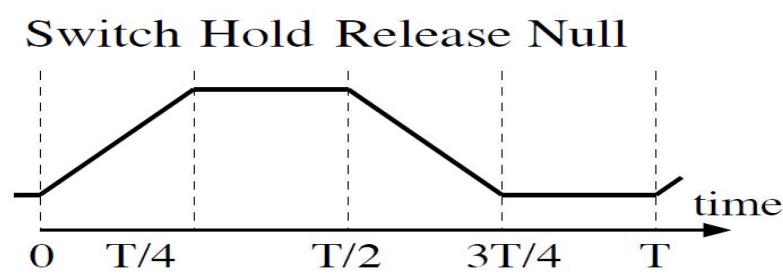

Figure 2. Phases of QCA Clock Scheme.

Another feature of QCA is that two perpendicular coplanar wires have the capability to interconnect with one another without the loss of signal as shown in Figure 3. In standard logic circuits, wire-crossing is the major issue for the successful transmission of information; the two wires are tangibly insulated by the two metallic wires which are separated by an insulator. In contrast, QCA wires can easily cross each other without interference. This has been possible by configuring the quantum dots in such a way that their fields do not interfere with each other. Most combinational and sequential QCA designs (counters ${ }^{\text {P-13, }}$, serial memories ${ }^{1}$, flip-flops, adders subtractors) use wire crossing techniques so as to reduce the area and time intricacies.

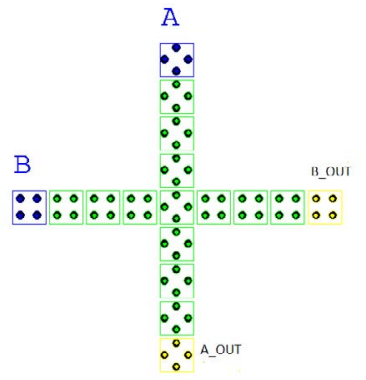

(a)

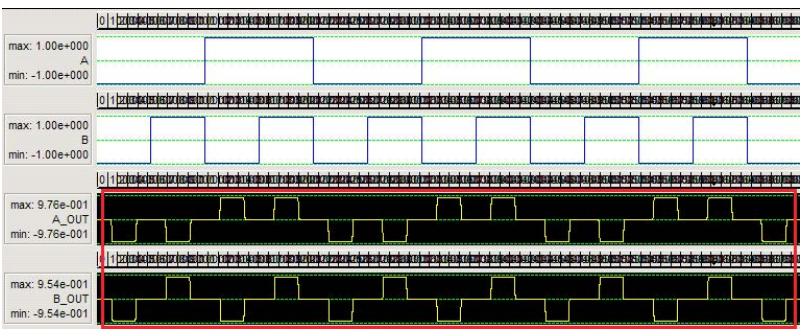

(b)

Figure 3. (a) Layout of the QCA Coplanar Crossover (b) Simulation Results of QCA Coplanar Crossover. 


\section{Related Work}

A wire crossing is a very important factor in QCA. Wire crossing methods are divided into three types as per the number of layers, cell state and clock phase mechanism: Co-planar ${ }^{6-18}$, multilayer ${ }^{19-21}$ and crossbar network methods $2-25$. Each of these methods has their own advantages but suffer from many problems of translation of cells, rotation of cells, time-dependent latching, and so on. A lot of work has been reported on wire crossing till date but unfortunately, a small amount of work has been reported on coplanar crossing with modular design in QCA. Some of the related works on different types of wire crossing are as under;

In 1 suggested coplanar wire crossing method in which horizontal and vertical wires are transferring the values of ' 0 ' and ' 1 ' respectively. Cells are rotated by $45^{\circ}$ in horizontal wire which induce the additional space between cells. When the number of cells in the vertical wire is sufficient after an intersection, a transferring value is not influenced by other wire. Due to the distinctive feature of rotated cells, the number of cells in the horizontal wire must be odd. The energy separation between the ground state and the first excited state decreases considerably with extra spacing between the cells. As a result of which the performance of the QCA circuits reduce in respect of maximum operating temperature, extremely low switching time and entropy resistance.

In 27 suggested a multilayer wire-crossing method which employs a crossover bridge system and is analogous to coplanar wire crossing because of the layout of the two crossing wires. There is no need of rotated cells in case of coplanar wire crossing. Therefore this method of wire crossing can be more generalized and miniaturized. Though this method has some advantages, but in the crossover area there occur noise problem between intersections of cells.

In f $^{3}$ suggested a crossbar network wire crossing method which is based on parallel to serial conversion where the cell arrangement assembly is regular. With the help of number of clocks or time dependent latching zones, the disadvantages of coplanar and multi layered wire crossing methods has been overwhelmed. If the clocking is constantly conserved by time-dependent latching, unexpected outcomes can arose. The outcomes can be switching time difficulty, cell temperature increase and kink energy noise. Likewise, this method involves two extra devices viz., a shift register and a parallel-toserial converter.

In ${ }^{2}$ suggested the new wire crossing method using the principle of the relation between relaxed and locked static stages. Even though the number of clock stages is bigger to its previous wire-crossings, still this method is having the following shortcomings:

- The design is not efficient as robustness lacks

- Alternate clocking is needed which is difficult to understand and manufacture; and

- Increase in the number of coplanar wires decreases the output polarity.

Therefore, it becomes necessary to propose a technique for designing robust coplanar crossing with special modular approach in QCA.

In this paper, three structures namely $4 \times 4,5 \times 5$ and $8 \times 8$ coplanar structures with unique modular design have been presented and the operation of these structures has been tested by QCA Designer Simulation Tool. It has been concluded that it is easy to increase the number of inputs by adding the suitable layers with proposed unique modular approach. The proposed modular coplanar structures allow number of coplanar wires to cross without signal degradation and signal loss until information propagates the output. Compared to the reported literature, the proposed design has following advantages;

- Unique modular approach in which wires can be intersected on the single layer without any intrusion or crosstalk

- High output polarity, fault tolerant capabilities, modular approach and robustness which makes it more efficient;

- Use of sequential clock which makes it easier to understand and manufacture;

- Does not have any kind of interference and effect on output polarity when the number of coplanar wires increases.

- The signal and its counterpart can be simply taken out together due to the alternating polarization.

- Proper use of clocking phase pairs in a traversed manner so as to adjust the required clock phases adjacent to each other, in order to permit the accurate propagation of signals.

- Cells are evenly disseminated into the clocking zones which are implemented in such a manner that the 
unusable spaces can be as small as feasible.

The above advantages can be understood by the following circuits designed in QCA.

\section{Coplanar Crossing}

Quantum-dot Cellular Automata (QCA) is a type of nanotechnology which offers special architectural design with two coplanar vertical wires having the capability to overlap with one another without the loss of information. These Vertical QCA wires can be employed by rotating the cells at 45 degrees by virtue of an inverter chain as shown in Figure 3. Wire Crossing can be achieved by intersecting either vertical or horizontalwire; these intersections are called as Cuts. Signal switching can be attained by four phases of clocking scheme. One of the most noticeable advantages of QCA as stated earlier is Coplanar Crossing because it permits single-layered circuit interconnection, whereas a different level of metallization is required in CMOS technology.

As far as a previous technical text is concerned, layouts have been considered in a particular clocking zone and outputs have been calculated by quasi adiabatic switching. A method proposed horizontal and vertical wires which are alternatively passing through an intersection. This method has an important feature of manipulating the pipelined function of QCA, single wire crossing which needs simple clocking and less area. Layouts familiarized for coplanar crossing in worked as follows: a) Normal crossing, established on the orientation of cells; b) Triple-Modular Redundant (TMR) crossing, established on the voting identity in QCA layout; and c) Thick crossing,established on the contact between cells in adistended wire.

\section{Proposed Coplanar Crossing with Unique Modular Approach}

Three structures namely $4 \times 4,5 \times 5$ and $8 \times 8$ coplanar structures with a unique modular design have been presented as follows:

\section{$4.14 \times 4$ Modular Coplanar Crossing}

In this structure, if input " $\mathrm{a}$ " is 1 or 0 , then the output ' $\mathrm{A}$ ' is 1 or 0 respectively. Similarly, outputs ' $\mathrm{B}$ ', ' $\mathrm{C}$ ' and " $\mathrm{D}$ " have same outputs as that of the inputs "b", " $c$ " and "d" respectively. The design is made in such a way that if all the inputs $(a, b, c, d)$ are given simultaneously, then the desired outputs (A, B, C, D) are taken simultaneously without any signal loss and signal degradation. The $4 \mathrm{x} 4$ modular coplanar crossing structures have $108694.70 \mathrm{~nm}^{2}$ $=0.11 \mu \mathrm{m}^{2}$ area, 70 cells and the output polarity is more than 0.9 approximately. The most important feature of this design is that the clocking is given in unique modular approach (shown with arrows). This feature allows the easy extension to implement high order coplanar crossing structures in QCA. The proposed $4 \times 4$ modular coplanar crossing and its simulated results are given in Figure 4(a) and Figure 4(b) respectively.

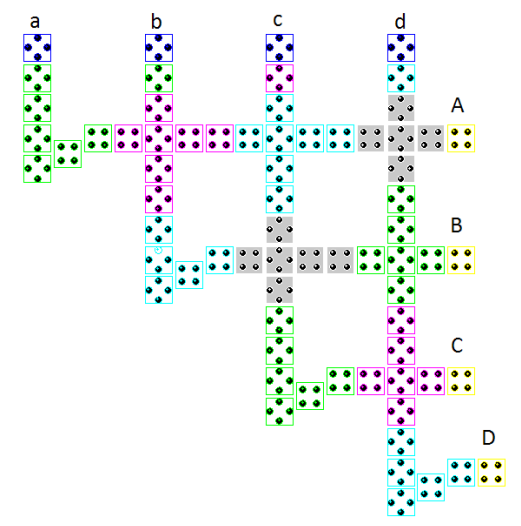

(a)

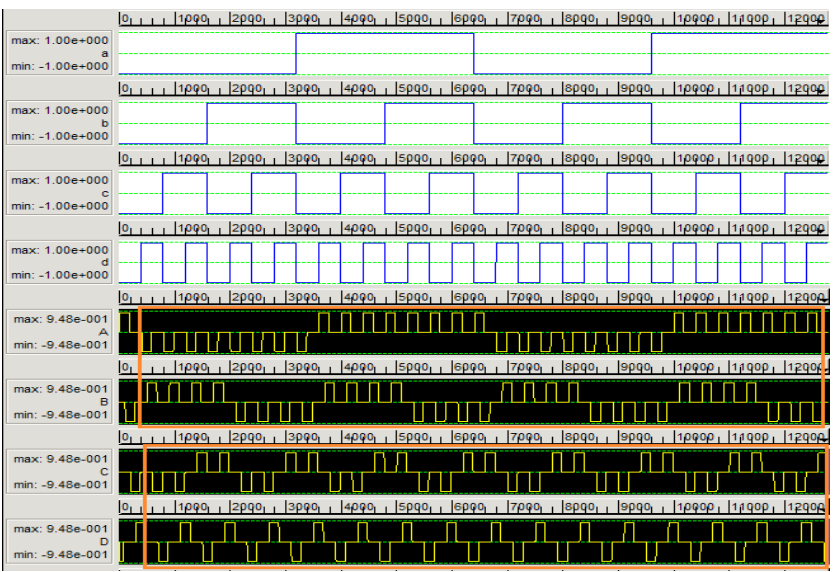

(b)

Figure 4. (a) Layout of $4 \times 4$ Modular Coplanar Crossing in QCA (b) Simulation Result of $4 \times 4$ Modular Coplanar Crossing.

\section{$4.25 \times 5$ and $8 \times 8$ Modular Coplanar Crossing Structures}

The $5 \times 5$ and $8 \times 8$ modular coplanar crossing structures are given in Figure 5(a) and Figure 5(b) respectively and 
the simulation results of $5 \times 5$ and $8 \times 8$ modular coplanar crossing structures are given in Figure 6(a) and Figure 6 (b) respectively. The area of $5 \times 5$ modular coplanar crossing design is $175839.28 \mathrm{~nm}^{2}=0.18 \mu \mathrm{m}^{2}$ while that of $8 \times 8$ modular coplanar crossing design is 421356.88 $\mathrm{nm}^{2}=0.42 \mu \mathrm{m}^{2}$. The $5 \times 5$ and $8 \times 8$ modular coplanar crossing structures contain 107 and 252 cells respectively. The output polarity in the both cases is more than 0.9 approximately.

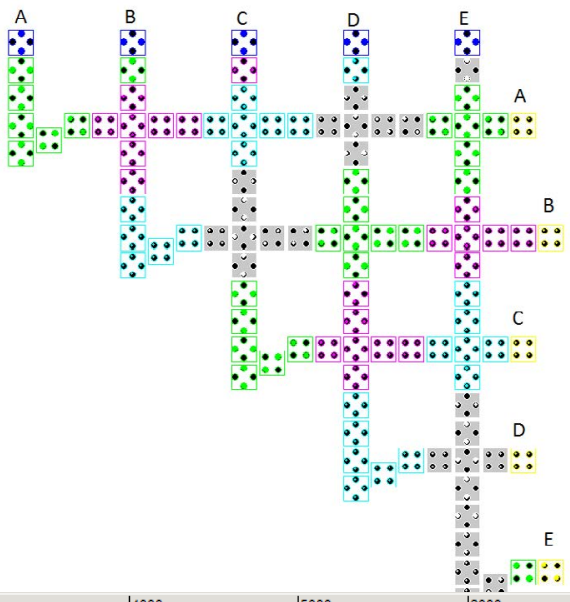

(b)

Figure 5. (a) Layout of 5x5 Modular Coplanar Crossing in QCA (b) Layout of 8x8 Modular Coplanar Crossing in QCA.

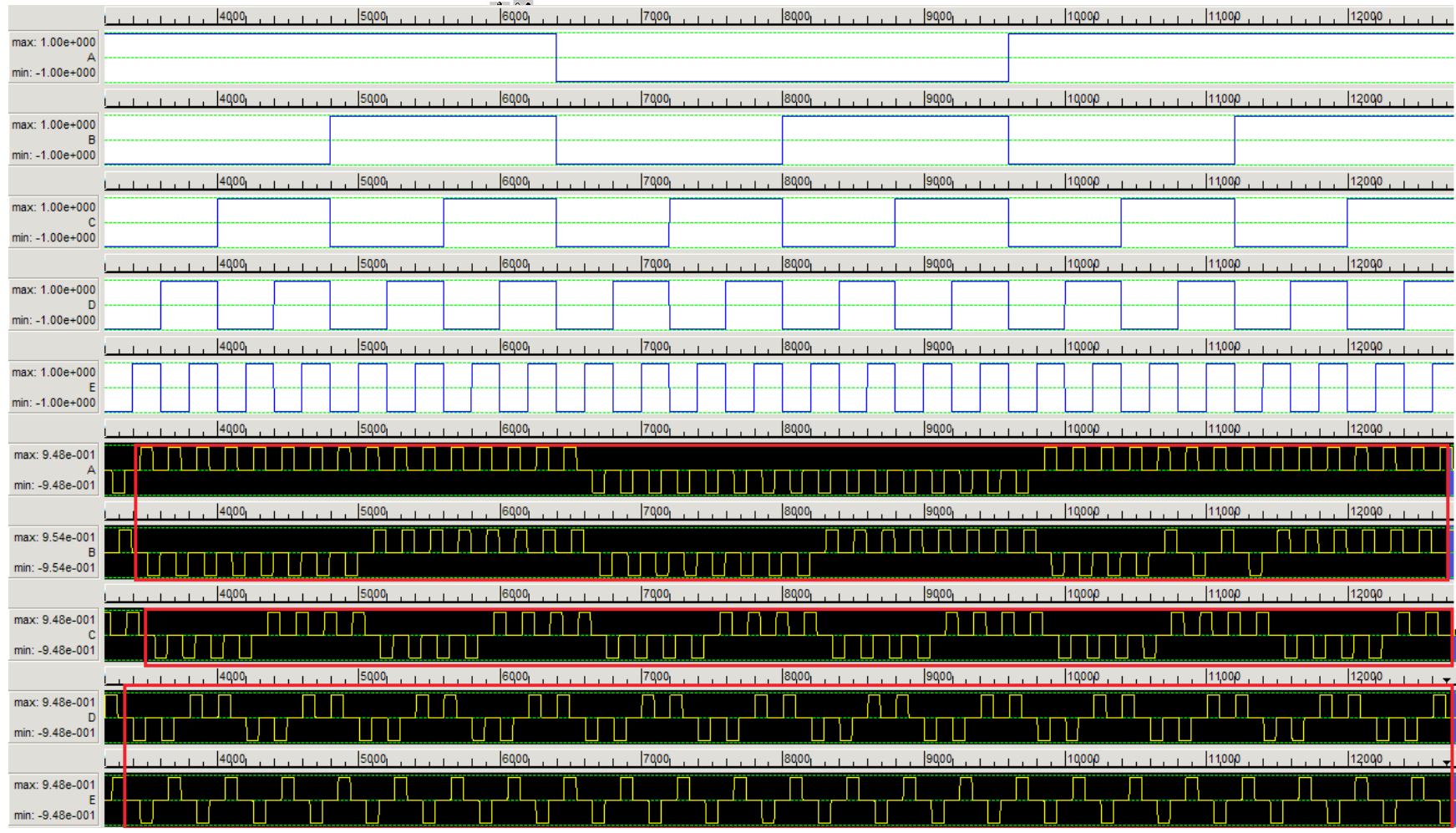

(a) 


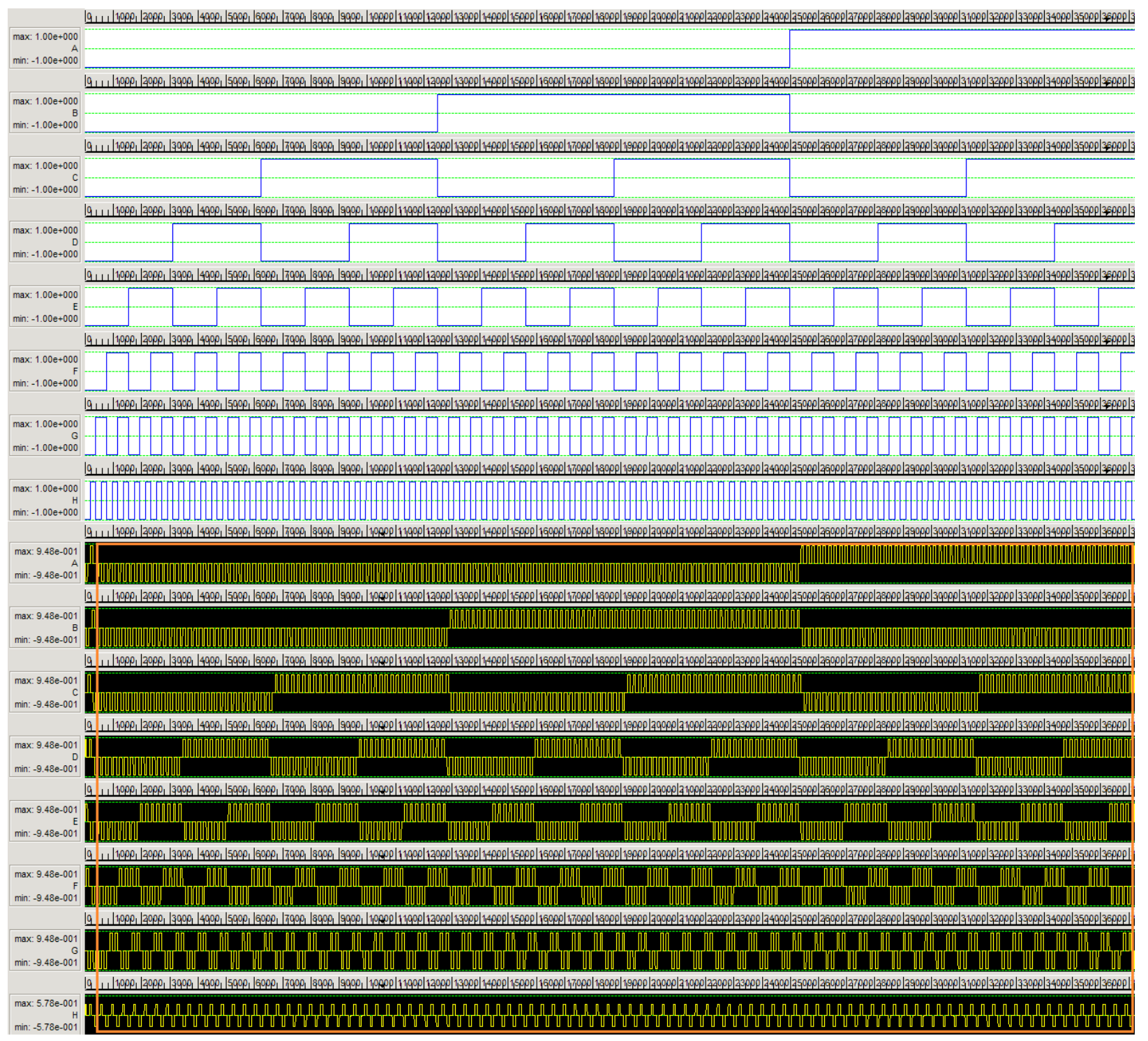

(b)

Figure 6. (a) Simulation Results of $5 \times 5$ Modular Coplanar Crossing (b) Simulation results of 8x8 Modular Coplanar Crossing.

The proposed $5 \times 5$ and $8 \times 8$ modular coplanar crossing designs can be transformed into thick designs as well which have the advantage of enhanced robustness. So as todetermine it, the thick version of the proposed $8 \times 8$ robust modular coplanar crossing and its simulation results has been presented in Figure 7(a) and Figure 7 (b) respectively. The $8 \times 8$ full robust modular coplanar crossing design has $671228.37 \mathrm{~nm}^{2}=0.67 \mu \mathrm{m}^{2}$ area. The output polarity and the number of cells are same as that of 8x8 modular coplanar crossing designs.

Further, from the coplanar crossing designs presented in Figure 4 and 5, it can be concluded that it is easy to extend the proposed modular coplanar crossing to larger
$\mathrm{N} \times \mathrm{N}$ modular coplanar crossing design by adding suitable number of layers to the preceding design.

The modular robust coplanar crossing presented in this paper proves efficient and robust as compared to conventional crossing designs of QCA. It is because of the modular approach which helps in extending the proposed design to $\mathrm{N}$ inputs making it more reliable than the conventional designs. In particular, if any of the cells is missing or misaligned, still the structures will give us the ate various faults during the manufacturing process.

In order to make proposed bus robust and more fault tolerant, it is necessary to increase the number of cells or wires shown in Figure 7(a). This is due to the fact that if 


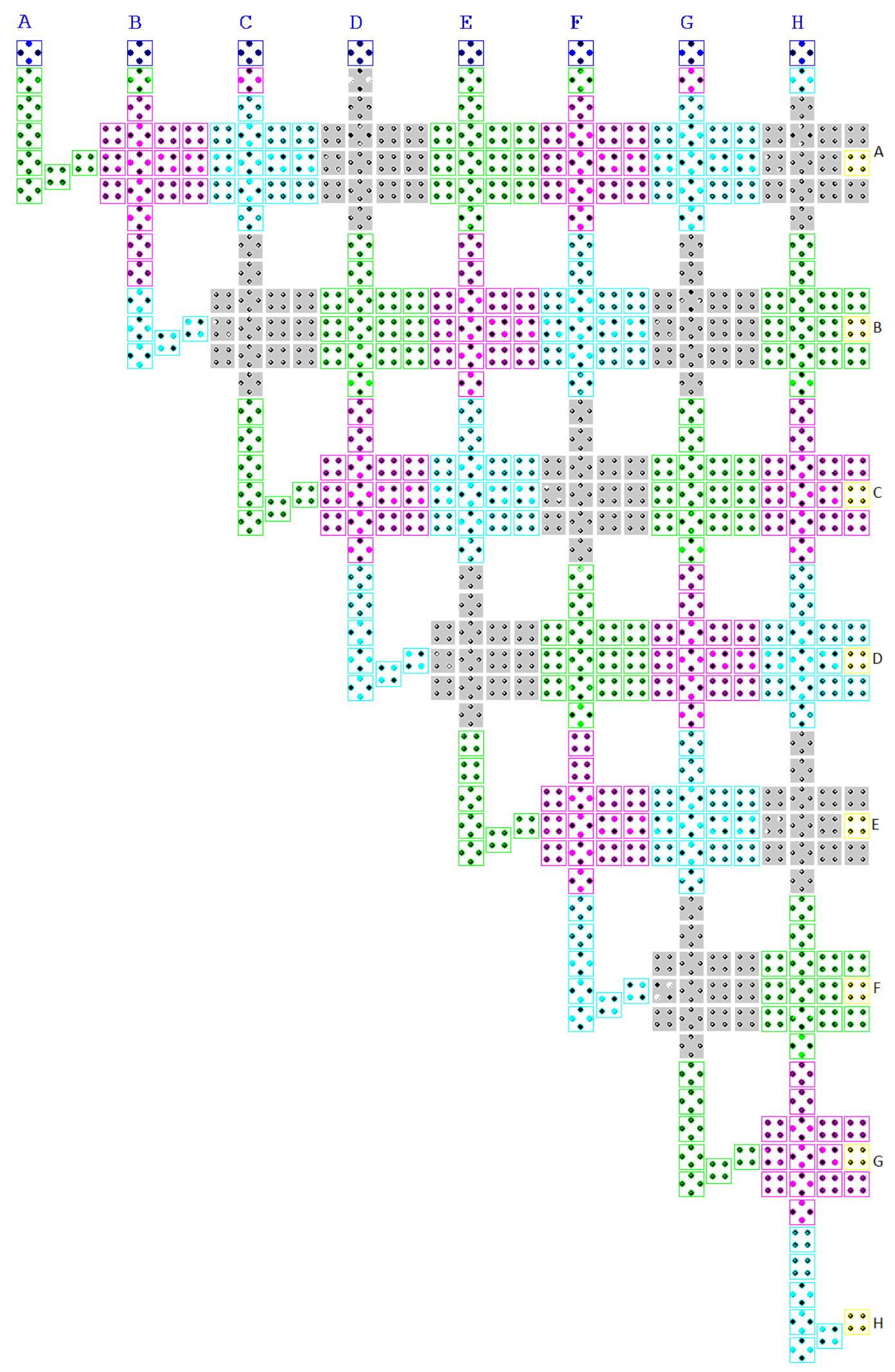

(a) 


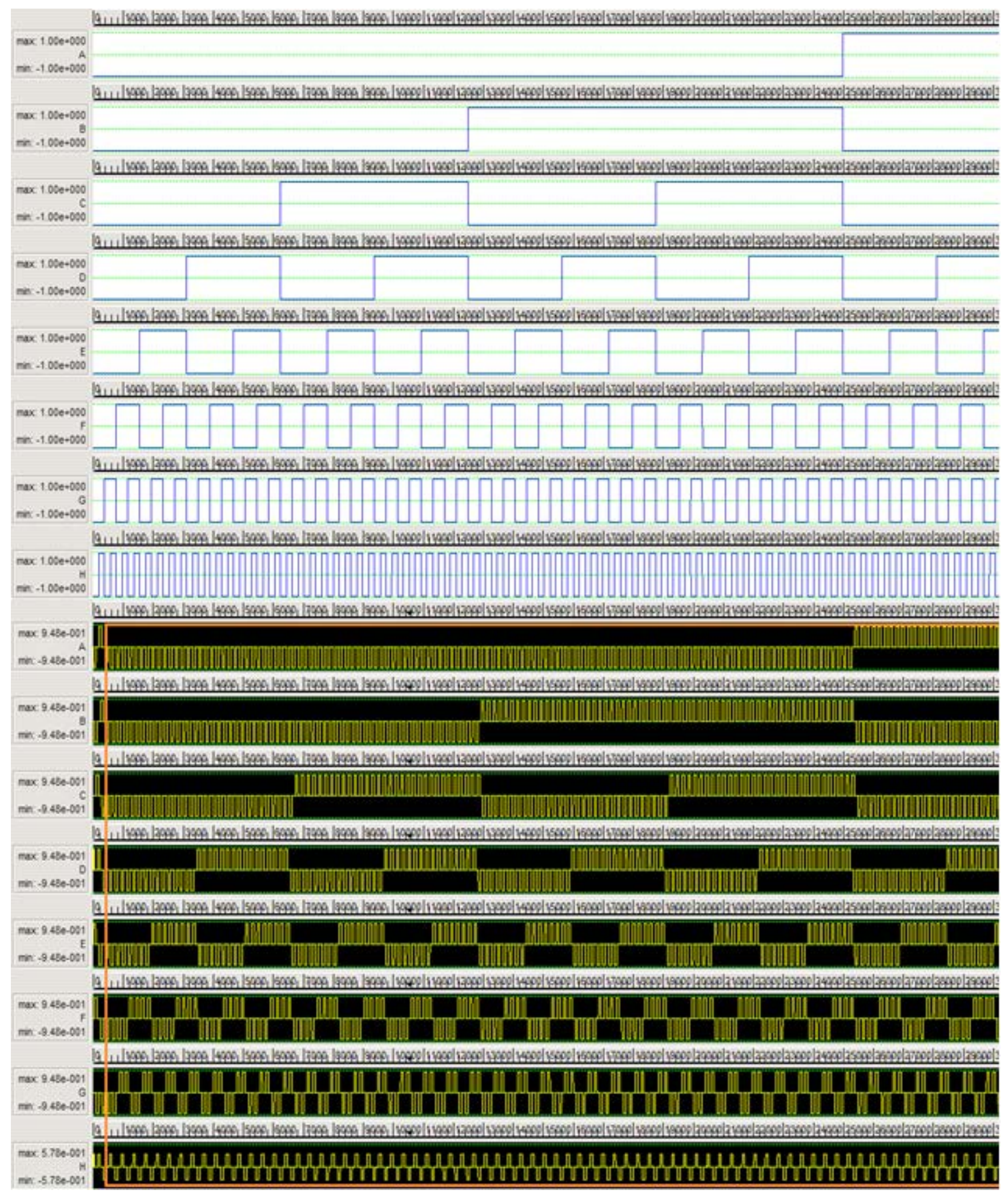

(b)

Figure 7. (a) Layout of 8x8 Robust Modular Coplanar Crossing in QCA (b) Simulation Result of 8x8 Robust Modular Coplanar Crossing. 
one of the cell is either misaligned or defective, the other wire or cell may proceed the input and the desired output will be taken.

\section{Conclusion}

This paper presents novel robust coplanar crossing with unique modular designs. Starting from the lowest number of inputs, it is easy to extend the proposed designs to large and then larger number of inputs by adding the suitable layers. Both normal and thick modular coplanar crossing designs have been presented. The proposed coplanar crossing designs will prove efficient to tolerate various faults of missing cell or misalignment during the manufacturing process. The operation of the proposed designs has been verified by the QCA Designer simulation tool.

\section{References}

1. Lent CS, Tougaw PD, Porod W, Bernstein GH. Quantum Cellular Automata. Nanotechnology.1993 January; 4 (1):49-57. Crossref

2. Lent CS, Tougaw PD. Lines of interacting quantum-dot cells: A binary wire. Journal of Applied Physics. 1993 August; 74(10):6227-33. Crossref

3. Amlani I, Orlov AO, Kummamuru RK, Bernstein GH, Lent CS, Snider GL. Experimental demonstration of a leadless quantum-dot cellular automata cell. Applied Physics Letters. 2000 July; 77(5):738-40. Crossref

4. Lent CS, Tougaw PD, Porod W. Quantum cellular automata based efficient BCD adder structure. Proceedings of the Workshop on Physics and Computing, IEEE Computer Society Press: DallasTX. 1994; 75:1818-25.

5. Amlani I, OrlovAO, Bernstein GH, Lent CS, Snider GL. Realization of a functional cell for quantum-dot cellular automata. Science. 1997 August, 227(5328):928-30.

6. Meurer B, Heitmann D, Ploog K. Excitation of three dimensional quantum dots. Physical Review B. 1993 October; 48(15):11488-491. Crossref

7. Amlani I, Orlov AO, Toth G, Bernstein GH, Lent CS, Snider GL. Digital logic gate using quantum-dot cellular automata. Science. 1999 April; 284:289-91. Crossref PMid:10195887

8. Tahoori MB, Huang J, Momenzadeh M, Lombardi F. Testing of quantum cellular Automata. IEEE Transactions on Nanotechnology. 2004 December; 3(4):432-42. Crossref

9. Cho H, Swartzlander EE. Adder designs and analyses for quantum-dot cellular automata. IEEE Transactions on Nanotechnology. 2007; 6(3):374-83. Crossref

10. Vankamamidi V, Ottavi M, Lombardi F. A serial memory by quantum-dot cellular automata (QCA). IEEE Transactions on Computers. 2008 May; 57(5):606-18. Crossref

11. Yang X, Cai L, Zhao X, Zhang N. Design and simulation of sequential circuits in quantum-dot cellular automata: Falling edge-triggered Flip-flop and counter study. Microelectronics Journal. 2010 January; 41(1):56-63. Crossref

12. Shamsabadi AS, Ghahfarokhi BS, Zamanifar K, Movahedinia N. Applying inherent capabilities of quantum-dot cellular automata to design: D Flip-flop case study. Journal of systems architecture. 2009 March; 55(3):180-87. Crossref

13. Vankamamidi V, Ottavi M, Lombardi F. Two-dimensional schemes for clocking/timing of QCA circuits. IEEE Transactions on Computer-aided design of integrated circuits and systems. 2008 January; 27(1):34-44. Crossref

14. Navi K, Farazkish R, Sayedsalehi S, Azghadi M R. A new quantum-dot cellular automata full-adder. Microelectronics Journal. 2010 December; 41(12):820-26. Crossref

15. Tougaw PD, Lent CS. Logical Devices Implemented using Quantum Cellular Automata. Journal of Applied physics. 1994 February; 75(3):1818-25. Crossref

16. Walus K, Dysart TJ, Jullien GA, Budiman RA. QCADesigner: A rapid design and simulation tool for quantum-dot cellular automata. IEEE Transactions on Nanotechnology. 2004 March; 3(1):26-31. Crossref

17. Pudi V, Sridharan K. Efficient design of a hybrid adder in quantum-dot cellular Automata. IEEE Transactions on Very Large Scale Integration (VLSI) Systems. 2011 September; 19(9):1535-48.

18. Janez M, Pecar P, Mraz M. Layout Design of Manufacturable Quantum-dot Cellular Automata. Microelectronics Journal. 2012 July; 43(7)501-13. Crossref

19. Tougaw D, Khatun M. A scalable signal distribution network for quantum-dot cellular automata. IEEE Transactions on Nanotechnology. 2013 March; 12(2):215-24. Crossref

20. Graunke CR, Wheeler DI, Tougaw D, Will JD. Implementation of a Crossbar Network using Quantum-dot Cellular Automata. IEEE Transactions on Nanotechnology. 2005 July; 4(4):435-40. Crossref

21. Tougaw D. Johnson EW, Egley D. Programmable logic implemented using quantum-dot cellular automata. IEEE transaction on nanotechnology. 2012 July; 11(4):739-45. Crossref

22. Wood JD. Tougaw D. Matrix multiplication using quantum-dot cellular automata to implement conventional microelectronics. IEEE Transaction on Nanotechnology. 2011 September; 10(5):1036-42. Crossref

23. Shin SH, Jeon JC, Yoo KY. Design of Wire-Crossing Technique Based on Difference of Cell State in Quantum-Dot Cellular Automata. International Journal of Control and Automation. 2014; 7(4):153-164. Crossref

24. Lent CS, Isaksen B, Lieberman M. Molecular quantum-dot cellular automata. Journal of the American chemical society. 2003 January; 25(4):1056-63. Crossref PMid:12537505

25. Bhanja S, Ottavi M, Pontarelli S, Lombardi F. Novel designs for thermally robust coplanar crossing in QCA. Proceedings of the conference on Design, Automation and Test in Europe. 2006 March; p. 786-91. Crossref

26. Bhanja S, Ottavi M, Lombardi F, Pontarelli S. QCA Circuits for Robust Coplanar Crossing. Journal of Electronic Testing. 2007 January; 23(2-3):193-210. Crossref 